\title{
Procedimentos Legais e Protocolos para Utilização de Cadáveres no Ensino de Anatomia em Pernambuco
}

\author{
Legal Procedures and Protocols for Use \\ of Cadavers in Anatomy Courses in \\ Pernambuco, Brazil
}

Elizabeth Neves de Melo José Thadeu Pinheiro

PALAVRAS-CHAVE

- Cadáveres.

- Legislação

- Anatomia

KEY-WORDS

- Cadaver.

- Legislation.

- Anatomy.
Artigo recebido em: 01/04/2009 Reencaminhado em: 13/08/2009 Aprovado em: 14/09/2009

\section{RESUMO}

A aquisição de cadáveres para estudo e pesquisa vinha sendo feita tradicionalmente utilizando-se corpos não reclamados, sem grandes formalidades. Com a complexidade dos tempos atuais, o simples encaminhamento desses corpos às escolas de Medicina passou a ser temido com o receio de transgredir a lei. Isso desencadeou uma redução no número de cadáveres para as aulas de Anatomia. Esta realidade motivou o Departamento de Anatomia da Universidade Federal de Pernambuco, juntamente com outras instituições do Estado, a pesquisar mecanismos legais que solucionassem a escassez de cadáveres para estudo e pesquisa. A metodologia utilizada constou de reuniões de discussão e apresentação de protocolos de recebimento de cadáveres com base principalmente na legislação vigente. Os resultados obtidos foram: (a) publicação do Provimento 28/2008 da Corregedoria Geral de Justiça do Tribunal de Justiça do Estado de Pernambuco, que dispõe sobre o registro de óbito dos cadáveres destinados às escolas de Medicina para fins de ensino e pesquisa; (b) criação de protocolos para o aproveitamento de cadáveres no Estado de Pernambuco. Concluímos que o conhecimento das atribuições por parte de cada uma das instituições envolvidas facilitará o processo de aproveitamento dos cadáveres.

\section{ABSTRACT}

The acquisition of cadavers for research and teaching has traditionally relied on unclaimed corpses, without major formalities. With the complexity of modern times, the mere transfer of these corpses to medical schools has come to be feared on grounds that it may break some law. This has reduced the number of cadavers available for anatomy classes. The Department of Anatomy at the Federal University in Pernambuco, Brazil, together with other institutions from the State, responded by studying legal mechanisms to overcome the lack of cadavers for research and teaching. The methodology consisted of discussion meetings and presentation of protocols for receiving cadavers, based mainly on the prevailing Brazilian legislation. The results were: (a) publication of Provision 28/2008 by the Pernambuco State Court, ruling on death certificates for cadavers scheduled for transfer to schools of medicine for research and teaching and (b) the creation of protocols for the use of cadavers in the State of Pernambuco. We conclude that knowledge of the attributions of the various institutions involved in the process will facilitate the use of cadavers in research and teaching activities. 


\section{INTRODUÇÃO}

O conhecimento de Anatomia é essencial para o início da educação médica, e o conhecimento obtido por meio da dissecação de cadáveres humanos é parte indispensável na educação de profissionais da área de saúde ${ }^{1}$. Entretanto, na história da Anatomia, a obtenção de cadáveres sempre foi um grande problema².

$\mathrm{Na}$ Europa, os cadáveres de criminosos eram utilizados em estudos anatômicos. Posteriormente, foram substituídos por cadáveres não reclamados, e nos últimos 50 anos os corpos doados são a maior fonte de cadáveres para estudo. Estas mudanças vêm ocorrendo do século 12 até hoje².

$\mathrm{Na}$ Índia, o Anatomical Act, um Ato de Estado promulgado pelo Poder Legislativo em 1949, regulamenta o uso de cadáveres não reclamados em instituições médicas e de ensino, com o propósito de dissecações anatômicas ${ }^{1}$. Neste caso, a morte deve ocorrer em hospital ou em local público dentro de zona prescrita por instituição médica, e a polícia deve declarar um período de 48 horas após a morte sem reclamação do corpo ${ }^{3}$. O governo indiano também tem incentivado doações voluntárias de cadáveres, mediante a conscientização das pessoas sobre a importância desta ação para assegurar que não exista escassez de corpos em instituições médicas ${ }^{1,4}$.

No Japão, Estados Unidos e Alemanha, são mantidos sistemas de captação de cadáveres humanos, por meio da divulgação ao público do processo de doação de corpos encaminhados para estudo nos departamentos de Anatomia das universidades ${ }^{5}$.

No Brasil, existia uma tradição verbal, sem maiores formalidades, de utilizar corpos de indigentes e de mortos não reclamados pelas respectivas famílias ${ }^{6}$. Na prática, estes cadáveres eram entregues às escolas da área de saúde para estudo e ensino de anatomia humana ${ }^{7}$. Este procedimento ocorreu como se fosse a lei. Em 30 de novembro de 1992, foi editada a Lei Federal 8.501, que disciplina a destinação de cadáver não reclamado junto às autoridades públicas, para fins de ensino e pesquisa e dá outras providências ${ }^{8}$. Entretanto, dificuldades no cumprimento das normas levaram a Universidade Federal de São Paulo a formular uma consulta à Corregedoria Geral de Justiça do Estado de São Paulo em 1997, que resultou no Provimento/CG nº 16 , de 26 de setembro de $1997^{9}$. Segundo esse Provimento, a instituição de ensino interessada na utilização de cadáver não reclamado deverá requerer o assento do óbito ao cartório competente, apresentando declaração de óbito e providenciando a publicação de editais que noticiem o óbito em jornais de grande circulação. Após autorização judicial, a certidão de óbito será lavrada mencionando que o cadáver se encontra insepulto na instituição de ensino requerente ${ }^{8}$.
Na Universidade do Vale do Itajaí, em Santa Catarina, desde 1998, existem dois métodos de recebimento de cadáveres para estudo anatômico: via Instituto Geral de Perícias, subordinado às regras estabelecidas com a Secretaria de Segurança Pública do Estado de Santa Catarina; e via sistema de doações ${ }^{10}$.

No Departamento de Anatomia da Universidade Federal de Pernambuco (UFPE), os alunos do curso médico matriculados nas disciplinas de Anatomia deixaram de dissecar desde 1998, devido à redução do número de cadáveres. Esse Departamento recebeu apenas dois cadáveres em três anos (20062008), procedentes de doações em vida e da família. Nesse mesmo período, não foi recebido cadáver não reclamado. A dificuldade na entrada de cadáveres para estudo no Departamento de Anatomia da UFPE tem comprometido o aproveitamento dos alunos de todos os cursos das áreas de saúde e biológicas. Estes alunos têm aulas práticas com cadáveres dissecados várias vezes, alguns com objetivos práticos inexistentes, bem como com a utilização de peças plásticas e programas de anatomia virtual.

O desenvolvimento tecnológico tem proporcionado muitas facilidades no ensino de Anatomia, mas a utilização de cadáveres (dissecação e prossecção) ainda é um componente essencial no processo de ensino e aprendizagem de Anato$\operatorname{mia}^{2,11-13}$

O objetivo deste estudo foi descrever as vias de obtenção de corpos humanos para o estudo anatômico em escolas de Medicina no Estado de Pernambuco, com base na Lei Federal 8.501, bem como entender os mecanismos legais desse processo e as atribuições das instituições envolvidas na regularização do cadáver para estudo.

\section{METODOLOGIA}

A metodologia utilizada constou de reuniões de discussão e apresentação de protocolos de recebimento de cadáveres com base principalmente na Lei Federal 8.501 e no Provimento/CG nº 16/97 do Estado de São Paulo. Estas reuniões ocorreram, desde meados de 2007, no auditório da Epidemiologia da Secretaria Estadual de Saúde de Pernambuco (SES/PE), com a participação de representantes da Gerência de Monitoramento e Vigilância de Eventos Vitais/SES; do Sistema de Informação de Mortalidade (Secretaria Municipal de Saúde - Recife); do serviço de necrópoles da Empresa de Manutenção e Limpeza Urbana (Emlurb); da Universidade Federal de Pernambuco (UFPE); da Universidade de Pernambuco; da Universidade Federal do Vale do São Francisco (PE); da Faculdade de Boa Viagem - Instituto Materno-Infantil (Imip); e do Centro Acadêmico de Vitória de Santo Antão (PE). 
As reuniões resultaram em um protocolo de intenções apresentado à Corregedoria Geral de Justiça do Estado de Pernambuco para avaliação e pronunciamento.

\section{RESULTADOS E DISCUSSÕES}

\section{Origem dos cadáveres destinados a ensino e pesquisa}

De acordo com os parágrafos $2^{\circ}$ e $3^{\circ}$, inciso $2^{\circ}$, artigo $3^{\circ}$, da Lei $8.501^{8}$, apenas os cadáveres de morte natural devem ser encaminhados a estudo e pesquisa em escolas de Medicina. Cadáveres de morte natural, cuja causa básica do óbito é uma doença ou estado mórbido ${ }^{14}$, poderão ser destinados para estudo, visto que não haverá responsabilidade alheia a apurar. Os cadáveres de morte provocada por mecanismos violentos ou suspeitos não deverão ser destinados a estudo, visto que há necessidade de esclarecer as circunstâncias em que se deu o fato.

Entre os cadáveres de morte natural destinados a ensino e pesquisa foram identificados: cadáver não reclamado com declaração de óbito emitida pelo Serviço de Verificação de Óbitos; cadáver não reclamado com declaração de óbito emitida pelo hospital da rede pública onde ocorreu o óbito; cadáver doado em vida; cadáver doado pela família.

Por cadáver não reclamado entende-se aquele de pessoa não identificada ou identificada sem responsável (parentes ou representante legal). O cadáver de pessoa não identificada é aquele que não dispõe de qualquer documentação. Já o cadáver de pessoa identificada compreende aquele sobre o qual inexistem informações relativas a endereços de parentes ou representante legal, entretanto apresentam ou documento emitido pelas autoridades públicas (cédula de identidade, certificado de reservista, título de eleitor, carteira profissional) ou outro documento identificatório (carteira de identidade expedida por empresas e/ou quaisquer outros documentos que sirvam para estabelecer sua identidade) ${ }^{15}$.

\section{Registro de óbitos dos cadáveres destinados às escolas de Medicina no Estado de Pernambuco}

Em 20 de setembro de 2008, a Corregedoria Geral de Justiça do Tribunal de Justiça do Estado de Pernambuco publicou o Provimento $28 / 2008^{16}$, que dispõe sobre o registro de óbito dos cadáveres destinados às escolas de Medicina para fins de ensino e pesquisa científica. Esse provimento foi elaborado pelo desembargador José Fernandes de Lemos, em resposta ao protocolo de intenções apresentado à Corregedoria, que enfatizou a necessidade primordial de complementar as leis sobre a utilização de cadáveres para fins de estudo e pesquisa científica no Estado de Pernambuco.
Na elaboração desse Provimento, o desembargador José Fernandes de Lemos considerou a enorme importância social, religiosa e jurídica do cadáver, cujo destino normal e rotineiro é o sepultamento ou a cremação, bem como a necessidade imperiosa de as escolas de Medicina utilizarem cadáveres para fins de ensino e pesquisa científica, os quais devem estar revestidos de todo o respeito ético e moral, além do rígido respeito às normas jurídicas. Disposições normativas estabelecidas pela Lei $\mathrm{n}^{\circ} 8.501^{8}$ de 30 de novembro de 1992 regulamentam as condições em que os corpos podem ser destinados a estudos, mas essa lei não resolve todos os problemas práticos decorrentes do encaminhamento de corpos às instituições de ensino da Medicina. Entre estes problemas estão dificuldades encontradas pelos oficiais do registro civil para elaborar os assentos de óbitos de cadáveres que se destinam a essas escolas, além de ainda ser praticamente inexistente a regulamentação dos casos de doação post mortem do próprio corpo "com objetivo científico ou altruístico", nos termos do artigo 14 do Código Civil ${ }^{17}$.

No Estado de Pernambuco, a publicação do Provimento $28 / 2008^{16}$ complementou o dispositivo legal ${ }^{8}$ existente, regulamentando que cadáver proveniente de pessoa não reclamada, identificada ou não identificada, bem como cadáver procedente de doação em vida ou doação pela família podem ser utilizados para ensino e pesquisa após o cumprimento de todos os itens desse Provimento.

\section{Organogramas para aproveitamento de cadáveres não reclamados para estudo}

Os protocolos propostos para aproveitamento de cadáveres para estudo e pesquisa foram embasados na Lei Federal $8.501^{8}$, de 30 de novembro de 1992, e no Provimento 28/2008 ${ }^{16}$ do Tribunal de Justiça do Estado de Pernambuco - Corregedoria Geral de Justiça, publicado em 20 de setembro de 2008 .

As figuras 1, 2, 3 e 4 apresentam as etapas necessárias ao processo de regularização do aproveitamento de cadáveres de morte natural não reclamados, identificados ou não, cujo atestado de óbito seja emitido pelo Serviço de Verificação de Óbitos (SVO) ou pelo hospital da rede pública onde ocorreu o óbito, sendo ainda definidas as atribuições de cada instituição envolvida nesse processo. Entre essas instituições temos: Gerência de Monitoramento e Vigilância de Eventos Vitais (GMVEV) da Secretaria de Saúde do Estado de Pernambuco, SVO, hospitais da rede pública, Escola de Medicina do Estado de Pernambuco, Cartório de Registro Civil, funerária. 
Figura 1

Fluxograma para aproveitamento de cadáveres sem identificação e sem causa-morte conhecida.

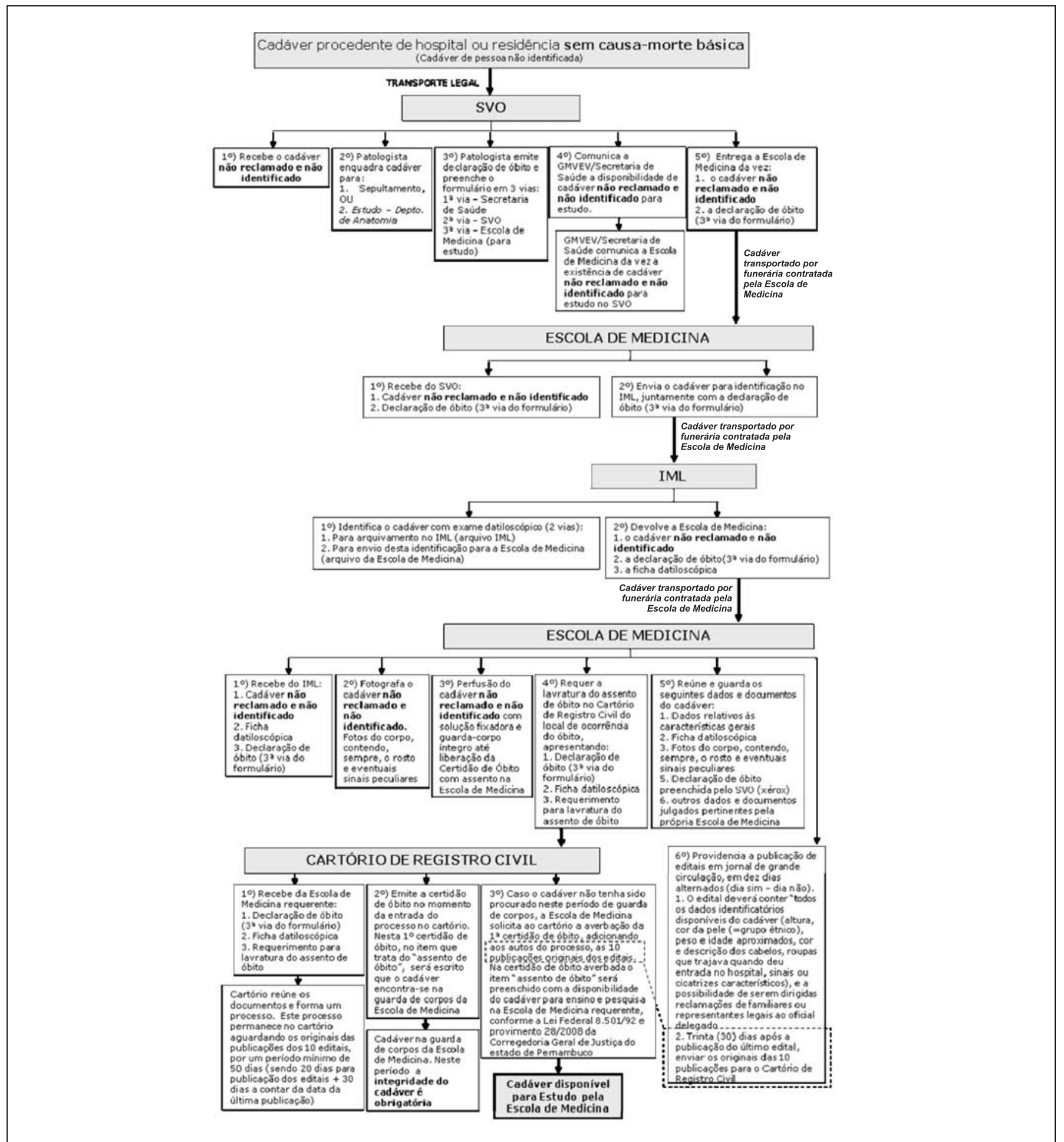

Etapas e atribuições de cada uma das instituições envolvidas no processo de regularização do aproveitamento de cadáver não reclamado e não identificado com declaração de óbito emitida pelo SVO. O direcionamento do cadáver para Escola de Medicina obedece a um rodízio controlado pela Gerência de Monitoramento e Vigilância de Eventos Vitais (GMVEV) da Secretaria de Saúde do Estado de Pernambuco. SVO — Serviço de Verificação de Óbitos. 
Figura 2

Fluxograma para aproveitamento de cadáveres com identificação e sem causa-morte conhecida.

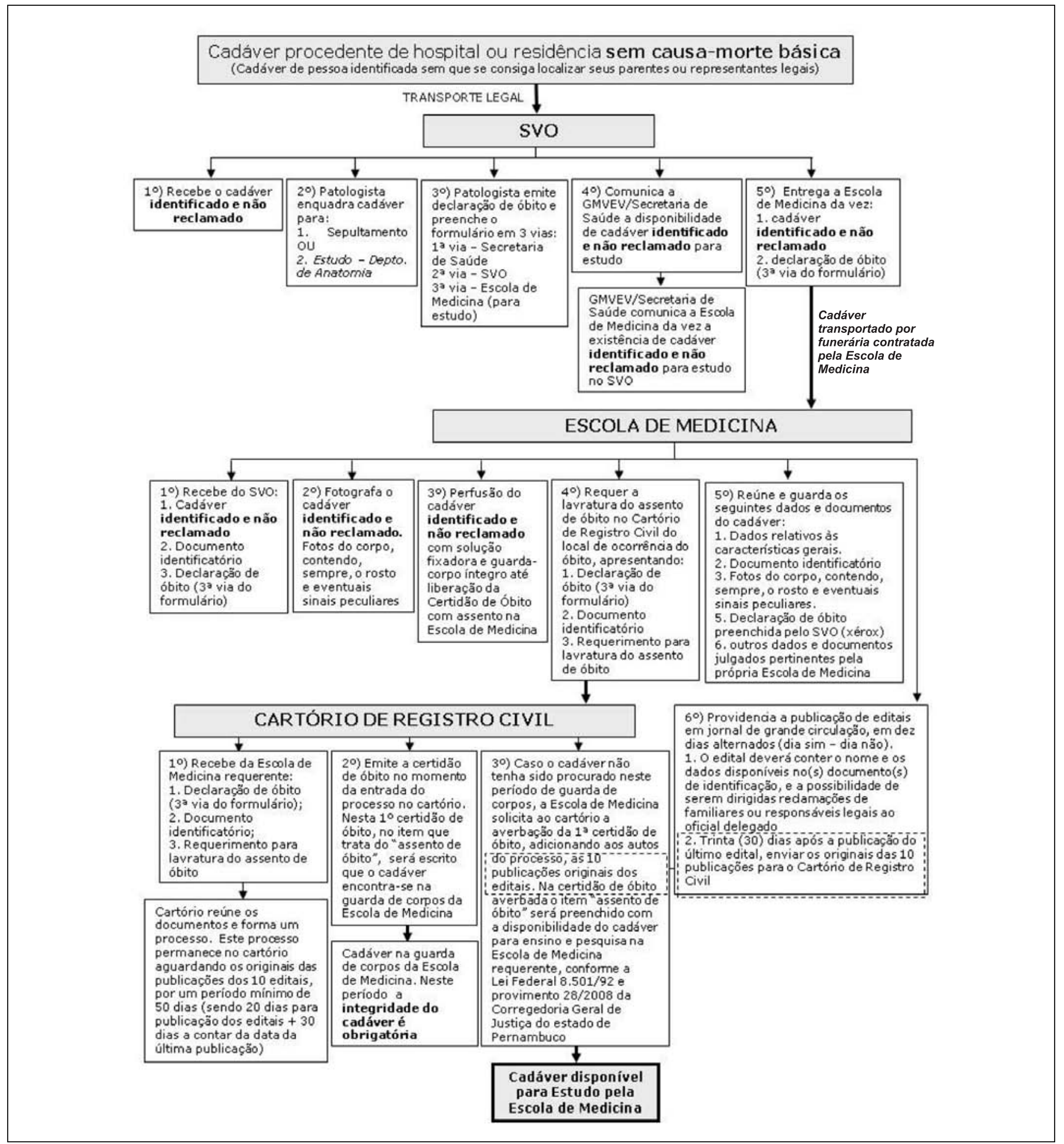

Etapas e atribuições de cada uma das instituições envolvidas no processo de regularização do aproveitamento de cadáver não reclamado e identificado com declaração de óbito emitida pelo SVO. O direcionamento do cadáver para Escola de Medicina obedece a um rodízio controlado pela Gerência de Monitoramento e Vigilância de Eventos Vitais (GMVEV) da Secretaria de Saúde do Estado de Pernambuco. SVO — Serviço de Verificação de Óbitos. 
Figura 3

Fluxograma para aproveitamento de cadáveres sem identificação e com causa-morte conhecida.

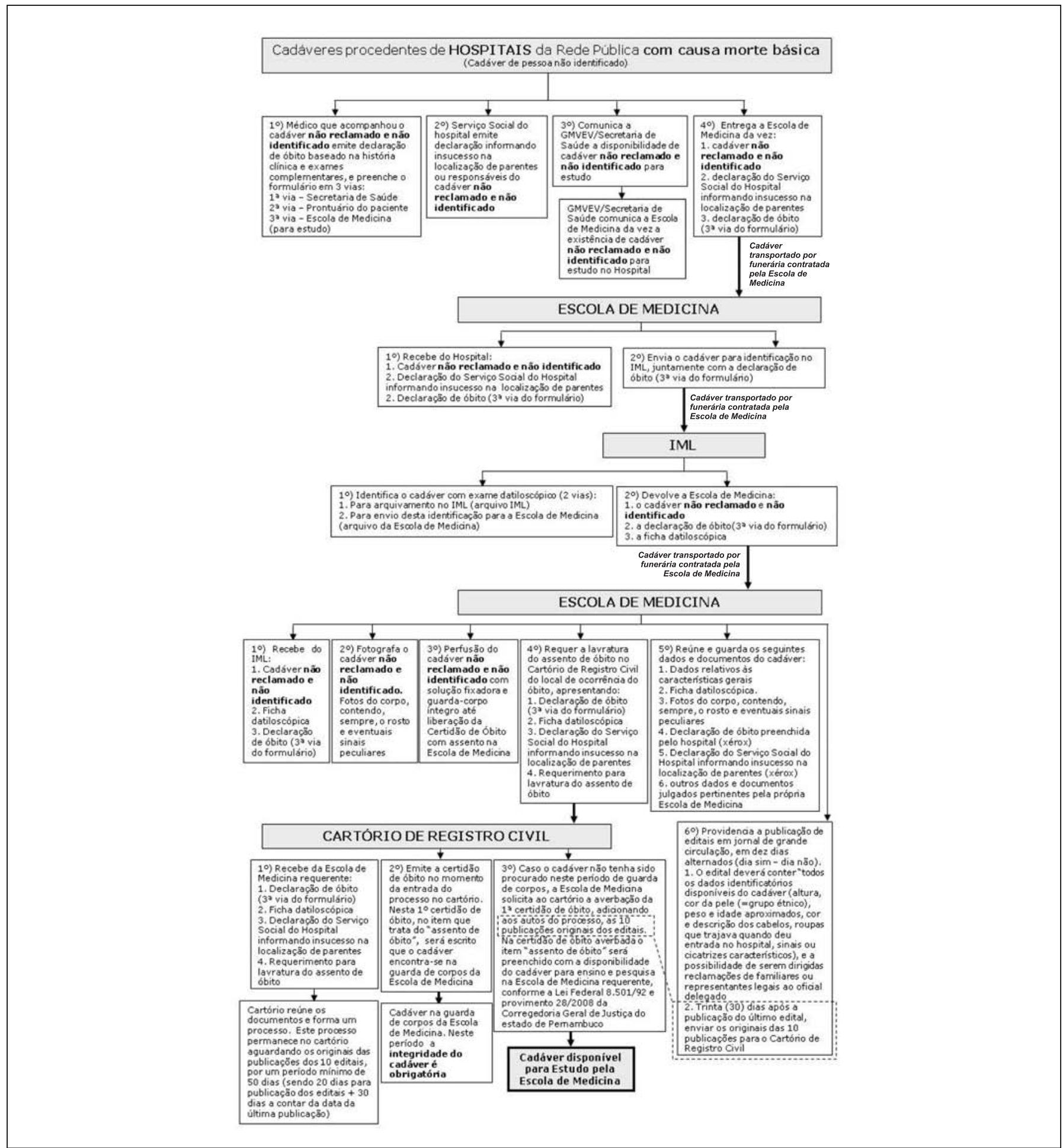

Etapas e atribuições de cada uma das instituições envolvidas no processo de regularização do aproveitamento de cadáver não reclamado e não identificado com declaração de óbito emitida pelo hospital da rede pública onde ocorreu o óbito. O direcionamento do cadáver para Escola de Medicina obedece a um rodízio controlado pela Gerência de Monitoramento e Vigilância de Eventos Vitais (GMVEV) da Secretaria de Saúde do Estado de Pernambuco. SVO — Serviço de Verificação de Óbitos. 
Figura 4

Fluxograma para aproveitamento de cadáveres com identificação e com causa-morte conhecida.

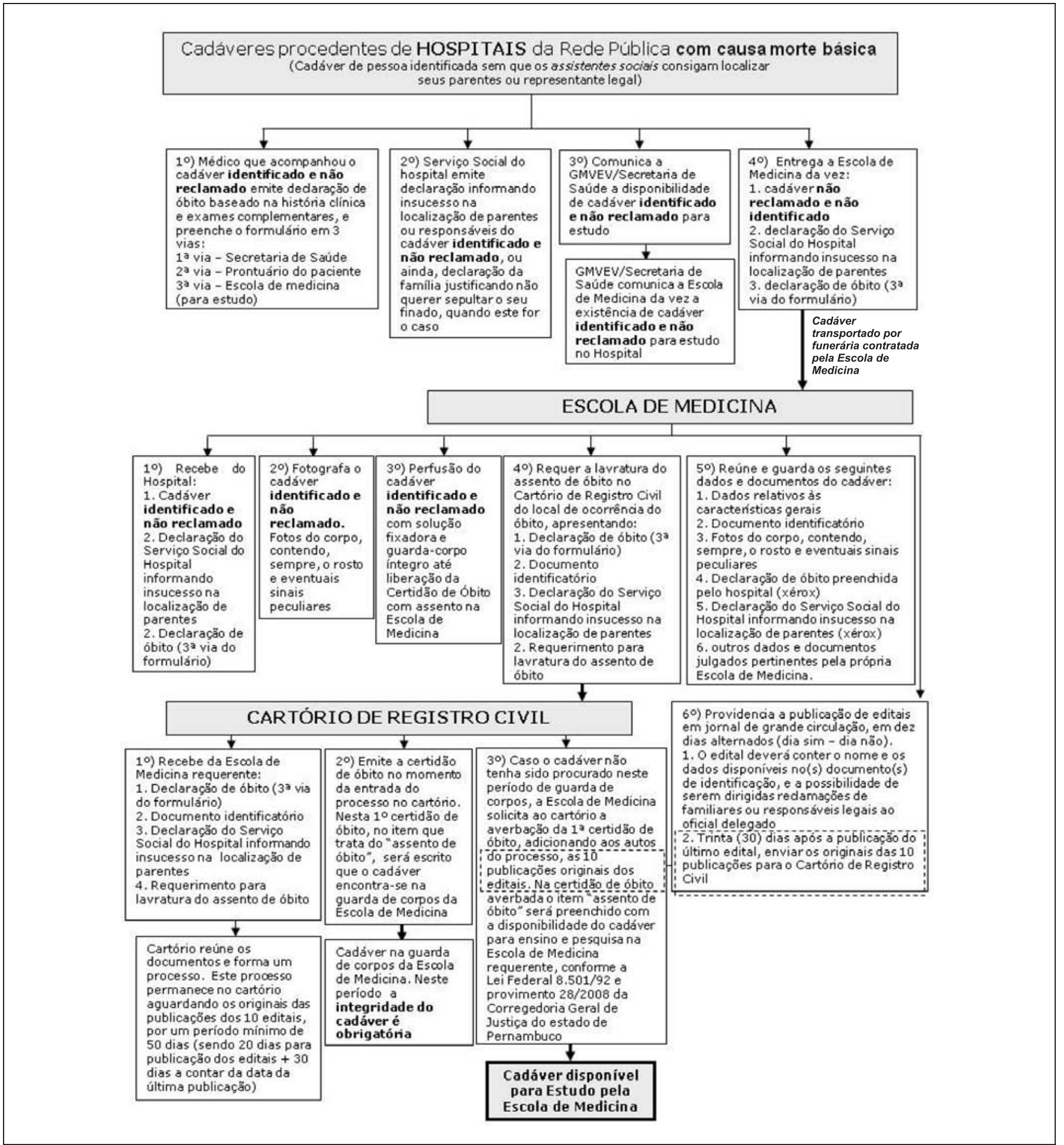

Etapas e atribuições de cada uma das instituições envolvidas no processo de regularização do aproveitamento de cadáver não reclamado e identificado com declaração de óbito emitida pelo hospital da rede pública onde ocorreu o óbito. O direcionamento do cadáver para Escola de Medicina obedece a um rodízio controlado pela Gerência de Monitoramento e Vigilância de Eventos Vitais (GMVEV) da Secretaria de Saúde do Estado de Pernambuco. SVO - Serviço de Verificação de Óbitos 


\section{Aproveitamento de cadáveres doados em vida ou pela família}

No Estado de Pernambuco, o aproveitamento de cadáveres doados em vida ou pela família está regido e regularizado com base no artigo 14 do Código Civil ${ }^{17}$ e no Provimento 28/2008 $(20 / 09 / 2008)^{16}$.

Na doação em vida, o doador emite uma declaração assinada por ele e duas testemunhas, todas com firma reconhecida em cartório, declarando que, em pleno gozo de suas faculdades mentais, deseja fazer doação espontânea do seu corpo após falecimento, para fins de estudo e pesquisa.

Para o cadáver doado pela família, o familiar ou representante legal do finado emite declaração que contemple o desejo de fazer doação espontânea do cadáver de seu parente, para fins de estudo e pesquisa. A declaração deve ter firma reconhecida.

Na declaração de doação em vida ou doação pela família, deve-se especificar a escola de Medicina para onde o cadáver será encaminhado.

Para receber o cadáver doado, a escola de Medicina, por intermédio de seu responsável legal, manifestará por escrito o interesse em recebê-lo e assumirá todas as responsabilidades legais, inclusive a de comunicar ao cartório, para fins de averbação no respectivo assento, e à família o término do interesse na utilização do corpo para fins de ensino e pesquisa de caráter científico. Este documento de manifesto será endereçado ao oficial do registro civil competente, juntamente com a declaração de óbito e a declaração de doação, para emissão da certidão de óbito, na qual constará no assento de óbito a escola de Medicina para onde o cadáver será encaminhado.

\section{Destino dos restos cadavéricos provenientes dos cadáveres utilizados nas escolas de Medicina}

Para os restos cadavéricos provenientes de corpos doados, está previsto no parágrafo $2^{\mathrm{o}}$ do artigo $7^{\mathrm{o}}$ do Provimento $28 / 2008^{16}$ que a escola de Medicina comunicará ao cartório, para fins de averbação no respectivo assento, e à família o término de interesse na utilização do corpo para fins de ensino e pesquisa de caráter científico. O parágrafo $4^{\circ}$ do referido artigo cita que, se a família ou representantes legais do falecido, no prazo de 15 dias, não manifestarem intenção em proceder ao sepultamento dos restos cadavéricos, a escola de Medicina, às suas expensas, providenciará esse sepultamento ou cremação ${ }^{16}$.

O sepultamento dos restos cadavéricos procedentes de cadáveres não reclamados será responsabilidade da Empresa de Manutenção e Limpeza Urbana (Emlurb) da Prefeitura do Recife. Com a certidão de óbito averbada, a escola de Medicina comunica a Emlurb para proceder ao transporte e sepultamento dos restos cadavéricos do corpo não reclamado.

\section{Repercussão da falta de cadáveres para estudo junto à sociedade}

A rádio $\mathrm{CBN}$ e os jornais locais do Estado de Pernambuco tornaram pública a falta de cadáveres na Universidade Federal de Pernambuco, bem como a publicação do Provimento $28 / 2008^{16}$, de 20 de setembro de 2007, da Corregedoria Geral de Justiça ${ }^{18,19}$. Esta veiculação pública sensibilizou a população, de forma que houve uma procura do Departamento de Anatomia da UFPE no sentido de efetivarem doações do corpo em vida, com o objetivo de auxiliar a escassez de cadáveres após o óbito.

\section{CONCLUSÕES}

Após estudos realizados acerca das leis sobre cadáveres destinados a estudo e pesquisa, e a função de cada setor (escola de Medicina, Secretaria de Saúde do Estado de Pernambuco, Empresa de Manutenção e Limpeza Urbana da Prefeitura do Recife e Cartórios de Registro Civil) na legitimação desses cadáveres, foi possível criar organogramas funcionais para o recebimento de cadáveres pelas escolas de Medicina. Estes organogramas instruem os procedimentos desde a origem do cadáver e seus trâmites legais, até o sepultamento dos restos cadavéricos, para cada uma das instituições envolvidas. A regularização dos procedimentos de recebimento de cadáveres obtidos legalmente e registrados de forma idônea resultou em confiança por parte dos indivíduos da sociedade, estimulando a doação espontânea.

\section{AGRADECIMENTOS}

Agradecemos ao desembargador José Fernandes de Lemos, da Corregedoria Geral de Justiça do Tribunal de Justiça do Estado de Pernambuco, que trabalhou para a publicação do Provimento 28/2008. Agradecemos também todo o empenho do grupo de trabalho constituído por integrantes da Universidade Federal de Pernambuco, Serviço de Verificação de Óbitos, Universidade de Pernambuco, Escola Pernambucana de Medicina, Universidade do Vale do São Francisco, Secretaria de Saúde do Estado de Pernambuco, Secretaria Municipal de Saúde do Recife e Emlurb.

\section{REFERENCIAS}

1. Ajita R, Singh I. Body donation and its relevance in anatomy learning: a review. J Anat Soc India. 2007;56(1):44-7.

2. Sehirli US, Saka E, Sarikaya O. Attitudes of Turkish anatomist toward cadaver donation. Clin Anat. 2004;17:677-81.

3. Subrahmanyam BV. Law in relation to medical men. In: Subrahmanyam BV, ed. Modi's Medical Jurisprudence and Toxicology. 22 $2^{\text {rd }}$ ed. New Delhi: Butterworths; 1999. p.721-7. 
4. Pampilly VS. Cadavers for anatomical dissection. Indian J Med Ethics [periodico na Internet]. 2005 [acesso em 2009 jul 6];2(1):[aproximadamente 3p.]. Disponível em: http:/ / www.issuesinmedicalethics.org/131oa016.html

5. Watanabe IS. O ensino de anatomia humana: o dilema da escassez de cadáveres. [online]. [acesso em 10 maio 2006]. Disponível em: http://www.usp.br/jorusp/arquivo/1998/jusp424/manchet/rep_res/opiniao.html>

6. Espirito Santo AM et al. Uso de cadáveres no estudo de anatomia humana nas escolas da área de saúde. Rev Goiana Med. 1981;27(1/2):107-16.

7. França GV. Direito médico. 5a ed. São Paulo: Fundo Editorial Byk; 1992.

8. Brasil. Lei Federal no 8.501 de 30 de novembro de 1992. Dispõe sobre a utilização de cadáver não reclamado, para fins de estudo ou pesquisas cientificas e dá outras providências. Diário Oficial da República Federativa do Brasil. Brasília,1 dez. 1992; p. 16519.

9. São Paulo. Corregedoria Geral de Justiça. Tribunal de Justiça do Estado de São Paulo Provimento 16/1997, de 26 de setembro de 1997. Regulamenta a lavratura de assentos de óbitos quando destinados cadáveres a estudo ou pesquisas científicas, como previsto pela lei $\mathrm{n}^{\circ}$ 8.501/92, e adiciona ao item 100 do Capítulo XVII das Normas de Serviço da Corregedoria Geral de Justiça. Diário Oficial da União. 26 set. 1997; p. 41.

10. Sgrott EA et al. Organograma de chegada de cadáveres no Laboratório de Anatomia da Universidade do Vale do Itajaí UNIVALI respeitando a Lei 8.501. In: XXII Congresso Brasileiro de Anatomia, XXVII Congresso Chileno de Anatomia e VIII Congresso de Anatomia do Cone Sul, 2006, Florianópolis. XXII Congresso Brasileiro de Anatomia, XXVII Congresso Chileno de Anatomia e VIII Congresso de Anatomia do Cone Sul, 2006. v. 1. p. 501-501.

11. Captier G, Canovas F, Bonnel F. Le corps humain et línformatique comme outils pédagogiques de l'anatomia. Morphologie. 2005; 89:142-53.

12. Bay BH. Can computer-aided instruction effectively replace cadaver-based learning in the study of human anatomy? CDTL Brief. 2007;10(3): 6-7.

13. Vacher C, Delmas V. Faut-il encore des dissections en faculté de médicine? Are dissections in faculty of medicine still needed? Morphologie. 2009;93:6-8.
14. Brasil. Ministério da Saúde. Conselho Federal de Medicina. Centro Brasileiro de Classificação de Doenças. A Declaração de Óbito: documento necessário e importante. Brasília, DF; 2007. p.12.

15. Marrey Neto JA. O aproveitamento de cadáveres para estudo de Anatomia. Rev Tribunais. São Paulo. 2007; 858:48396

16. Pernambuco. Corregedoria Geral de Justiça. Tribunal de Justiça do Estado de Pernambuco. Provimento 28/2008 de 11 de setembro de 2008. Dispõe sobre o registro de óbito dos cadáveres destinados às Escolas de Medicina, para fins de ensino e pesquisas de caráter científico. Diário Oficial do Estado de Pernambuco. Poder Judiciário, de 20 set. 2008; p.5-6.

17. Brasil. Novo Código Civil. Lei no 10.406 de 10 de janeiro de 2002. Brasília, DF; 2002.

18. Sarmento W. "Precisa-se de Cadáveres". Jornal Comercio. 2008; 28 abr.: cidade 4.

19. Diário de Pernambuco. “UFPE está sem corpos para aulas de anatomia". 2008; 14 out.:vida urbana - C4.

\section{CONTRIBUIÇÃO DOS AUTORES}

Elizabeth Neves de Melo contribuiu na concepção e desenho do estudo, aquisição de dados, análise e interpretação dos dados, elaboração de versão inicial e final do artigo e na aprovação final da versão encaminhada para publicação

José Thadeu Pinheiro contribuiu substancialmente na concepção e desenho do estudo, análise e interpretação dos dados, revisão crítica do artigo e na aprovação final da versão encaminada para publicação

\section{CONFLITO DE INTERESSES}

Declarou não haver.

\section{ENDEREÇO PARA CORRESPONDÊNCIA}

Elizabeth Neves de Melo

Departamento de Anatomia - Centro de Ciências

Biológicas - UFPE

Av. Prof. Moraes Rego, s/n

Cidade Universitária - Recife

CEP 50670-420 - PE

E-mail: bethneves@hotmail.com 